\title{
Notas y comentarios
}

Nueva presencia de la mujer venezolana en el mercado de trabajo*

\section{Irene Casique**}

\section{Introducción}

Durante los últimos quince años se ha despertado, a nivel mundial, mayor sensibilidad e interés por la problemática de la incorporación de la mujer como elemento activo en la dinámica productiva de cada país, y en general, respecto al papel que debe jugar la mujer en el desarrollo de las naciones.

Esta sensibilidad ha quedado expresada a través de una amplia producción de textos y propuestas de investigación en torno al tema, en buen número de países, al igual que en la promoción y desarrollo de programas a favor de la mujer.

Sin embargo, aunque todas estas acciones, emprendidas por organismos gubernamentales y no gubernamentales, han sido fundamentales en la acumulación y profundización de conocimientos sobre la temática de la participación de la mujer, todavía persisten muchos de sus aspectos en un estado de conocimiento confuso y desarticulado.

Y de la misma manera, en la realidad cotidiana, los problemas fundamentales de segregación ocupacional, subcapacitación, subocupación, subremuneración y doble carga (trabajo-hogar) siguen afectando a la gran mayoría de las mujeres trabajadoras, especialmente en los países del tercer mundo.

En Venezuela, los aportes que se han hecho para obtener el conocimiento sobre la situación laboral resultan insuficientes y superficiales. Es así como encontramos que tanto el mercado de trabajo femenino venezolano, como las características de las tra-

* El presente artículo es un extracto del proyecto "Mujer y mercado de trabajo. Cambios recientes en la Región Capital de Venezuela", presentado en noviembre de 1991 como trabajo de grado para la maestría en demografía de El Colegio de México. Las limitaciones de espacio para la publicación de este artículo imponen una apretada síntesis del mismo; sin embargo, creemos que logra presentar los principales aspectos abordados en dicho trabajo.

** Profesora-investigadora del Departamento de Estudios Demográficos del Instituto de Investigaciones Económicas y Sociales de la Universidad Católica Andrés Bello. 
bajadoras, y las condiciones que rodean su inserción y desem peño laboral han sido objeto de muy pocos estudios sistemáticos. Buena parte de lo que creemos saber al respecto se origina en impresiones y lugares comunes, sin mayor fundamento, o en información no actualizada. Tal situación es no sólo atribuible a la falta de continuidad en las iniciativas planteadas, sino también, en gran medida, a la carencia de estadísticas e indicadores adecuados para aproximarse a la realidad.

En ese contexto encuentra justificación y relevancia la insistencia en la reflexión sobre los aspectos que obstaculizan una incorporación plena y cabal de la mujer en el ámbito laboral.

El punto de partida de este artículo es la afirmación de que, al menos desde 1980, el proceso de incorporación de la mujer venezolana al mercado de trabajo ha estado fuertemente asociada a las nuevas condiciones que la crisis - no sólo económica - ha definido en América Latina, y en nuestro caso particular en Venezuela. Junto a ello, importantes cambios respecto al tamaño deseado de la familia, una significativa reducción de la fecundidad y culminación de la reproducción a una edad más temprana, redefinen nuevas circunstancias, que han conducido a un proceso de incorporación al mercado de trabajo, de mujeres que tradicionalmente, por sus características particulares, no participaban de manera significativa.

En efecto, si bien la participación de la mujer venezolana se mantuvo en crecimiento durante la década de los ochenta, este incremento podría parecer poco relevante si se considera sólo en términos del aumento global de la tasa de actividad. Más bien su particularidad descansa en las características de las mujeres que durante este periodo aumentaron su actividad laboral.

Ello a su vez supone la configuración de un nuevo "perfil" de la mujer en el mercado de trabajo, que si bien puede ser que no esté todavía claramente definido, ya evidencia cambios cualitativos que, en determinado momento, podrían significar modificaciones sustanciales sobre el tipo de actividades en las que la mujer participe.

Por todo esto planteamos aquí una revisión de algunos factores a sociados con la incorporación de la mujer al mercado laboral, así como algunos aspectos de su inserción, en una búsqueda de elementos que permitan apuntar cambios recientes en el perfil de la mujer trabajadora y en su esquema de participación.

Nuestra revisión se fundamenta en datos de la Encuesta de Hogares por Muestreo levantada por la OCEI (Oficina Central de Estadística e Informática) para el segundo semestre de 1990, que para el momento de realizar este trabajo era la única fuente dispo- 
nible; en la actualidad se cuenta con otras encuestas, muy recientes (la Encuesta Social y la Encuesta de Fecundidad de 1991), ${ }^{1}$ que si bien se encuentran todavía en fase de validación, ofrecerán nuevas alternativas para el enriquecimiento de investigaciones como ésta. Por otra parte, nuestro análisis se centra exclusivamente en la región capital de Venezuela que, al concentrar la mayoría de los servicios y las mejores oportunidades laborales, al tiempo de que es la región más densamente urbanizada del país, registra de manera más acelerada y definida los cambios en torno a la actividad económica femenina.

\section{Evolución reciente de algunos factores asociados a la participación laboral femenina}

A diferencia del hombre, la participación de la mujer en el mercado de trabajo ha quedado determinada, tradicionalmente, no sólo por sus características personales, tales como edad y educación, y por las condiciones específicas del mercado de trabajo, sino también por una serie de condiciones familiares y por un conjunto de pautas y valores sociales que delinean, todos ellos, la definición de un papel femenino en la fuerza de trabajo, con características y dinámica particulares.

A estos factores asociados a la participación femenina nos queremos referir en este capítulo, con la presunción de que su conocimiento, como elementos que condicionan las posibilidades de participación de la mujer en el mercado de trabajo, es indispensable para una cabal comprensión de las características y significado de la actividad laboral femenina, y por ende para la formulación de políticas y la promoción de aquellos cambios que aparezcan como necesarios o deseables en una estructura social determinada.

Los factores asociados a la participación femenina, comúnmente llamados factores determinantes, suelen ser clasificados como individuales y sociales (Elizaga, 1977); complejizando un poco más el espectro, se pueden distinguir tres tipos de factores: contextuales, familiares e individuales (Christenson, García y De Oliveira, 1989).

En este artículo nos limitamos a revisar sólo tres factores, a saber: edad y nivel educativo de la mujer, como factores indivi-

\footnotetext{
${ }^{1}$ Levantadas el año pasado, respectivamente, por la Oficina Central de Estadística e Informática y por el Departamento de Estudios Demográficos del Instituto de Investigaciones Económicas y Sociales de la Universidad Católica Andrés Bello.
} 
duales, y su estado conyugal, como factor familiar. La selección de los tres aspectos se funda en las posibilidades de información que la fuente proporciona, en este caso la Encuesta de Hogares por Muestreo.

Cabe señalar que aunque nuestra revisión se centra en las tres características ya señaladas, su análisis debe hacerse contextualizándolas en el conjunto más global de elementos que conforman una realidad integrada. Así, este primer acercamiento, más que ofrecer respuestas y constituir un capítulo cerrado, sugiere múltiples preguntas y vetas por explorar en torno al tema.

\section{Actividad según la edad de la mujer}

Esta característica ha sido uno de los factores más estudiados tradicionalmente. Para una apreciación justa de su significado es necesario no perder de vista que la edad de la mujer va asociada, de manera estrecha, con cambios en el estado civil y con las etapas del ciclo familiar. Los cambios que involucra esta variable determinan, con frecuencia, marcadas diferencias en las tasas de participación femenina, en función del grupo de edad que se considere (Pantelides, 1976; Recchini y Wainerman, 1981, entre otros).

Tradicionalmente, los estudios realizados tanto en países desarrollados como en subdesarrollados, apuntaban una mayor participación de las mujeres más jóvenes. Ello parecía fácilmente explicable: las mujeres trabajaban, convencionalmente, hasta el momento en que se casaban o tenían al primer hijo, cuando se veían precisadas a retirarse completa o parcialmente para asumir las tareas del hogar. Este razonamiento, con el que nos conformamos por mucho tiempo, tiene en realidad poco alcance explicativo, ya que puede, de algún modo, dar cuenta de un tipo promedio de mujer clase media, pero no permite explicar situaciones distintas: ¿y qué pasa con las mujeres pobres?, ¿o con las mujeres solas?, ¿o con las que tienen expectativas de vida diferentes?

Sin embargo, investigaciones recientes, realizadas durante los ochenta en algunos países latinoamericanos, descubren modificaciones significativas de aquella "pauta tradicional" de asociación entre la edad y la participación laboral de las mujeres, al evidenciarse un repunte en el número de aquellas mayores de 25 años (García y De Oliveira, 1990).

Si revisamos esta situación en el caso de Venezuela, nos encontramos con que también aquí han venido modificando las pautas de participación por edad de la mujer. Tenemos así que, por lo menos hasta principios de los ochenta, eran las mujeres más jó- 
venes (menores de 25 años) las que registraban las mayores tasas de participación, al tiempo que la actividad iba disminuyendo progresivamente con la edad, en tanto que ahora se observa una disminución en la participación de las mujeres más jóvenes y un aumento de la actividad laboral de mujeres en edades medias.

El cuadro 1 nos permite apreciar estos cambios en las mujeres de la región capital. Al comparar las tasas de actividad femenina entre 1980 y 1990 , se observa una disminución del nivel por parte de las mujeres más jóvenes: entre 15-19 y 20-24 años, y un aumento de las tasas correspondientes a los demás grupos de edad. Para las mujeres más jóvenes, al igual que para los hombres, hay una disminución de la actividad económica; esto queda reflejado en la reducción de los valores de las tasas correspondientes a 15-19 y 20-24 años. Diversos autores coinciden en atribuir esto a una extensión de los años de escolaridad promedio (Hernández et al., 1982; Mazzei, 1986; Cartaya, 1986).

\section{CUADRO 1}

Venezuela, Región Capital. Tasas especificas de participación por sexo, según grupos de edad, 1980 y 1990

\begin{tabular}{lccccc}
\hline & \multicolumn{2}{c}{1980} & & \multicolumn{2}{c}{1990} \\
\cline { 2 - 3 } \cline { 5 - 6 } Grupo de Edad & Hombres & Mujeres & & Hombres & Mujeres \\
\hline $15-19$ & 32.69 & 17.84 & & 29.12 & 17.39 \\
$20-24$ & 72.10 & 43.99 & 71.12 & 41.60 \\
$25-34$ & 90.68 & 48.02 & 86.17 & 52.98 \\
$35-44$ & 95.24 & 46.03 & & 91.08 & 55.52 \\
$45-54$ & 92.21 & 36.88 & 88.58 & 43.27 \\
$55-64$ & 80.25 & 20.40 & 74.08 & 22.37 \\
65 y más & 45.39 & 7.22 & & 42.18 & 8.06 \\
\hline
\end{tabular}

Fuente: OCEI, Encuesta de Hogares por Muestreo, 1980 y 1990 , segundos semestres. Cálculos propios.

Las mujeres del grupo de 35 a 44 años registran incrementos especialmente importantes; su tasa de actividad pasa de 46.03 a 55.02. De tal manera que, mientras hacia 1980 la tasa de actividad más alta correspondía a las mujeres entre 25 y 34 años, en 1990 el máximo de la curva se desplaza al grupo de 35-44 años, como lo podemos observar en la gráfica 1.

Este aumento de las tasas de participación de las mujeres de mayor edad podría atribuirse, simultáneamente, a un retorno al trabajo de mujeres que ya culminaron su fase reproductiva y a una mayor permanencia de las mujeres en el trabajo, producto probablemente, de las condiciones de crisis prevalecientes, que 
GRÁFICA 1

Venezuela, Región Capital.

Tasas de actividad femenina, 1980 y 1990

Porcentaje

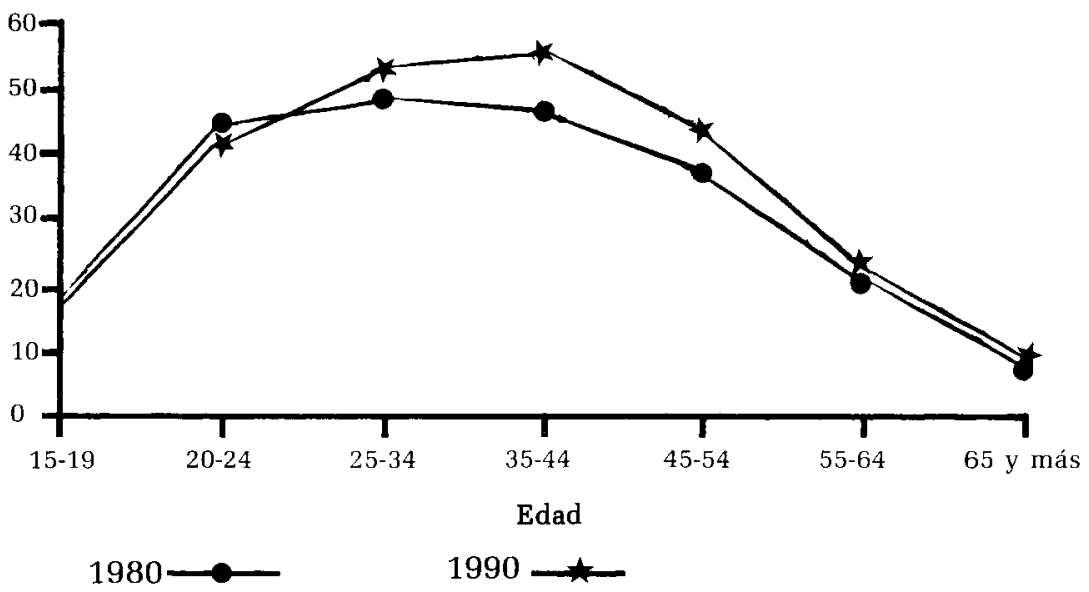

imponen para los hogares mayor necesidad del ingreso aportado por la mujer.

Frente al aumento de las tasas de actividad de las mujeres de todos los grupos de edad a partir de 25 años, se constata un proceso inverso para los hombres, en cuyo caso, durante el mismo periodo 1980-1990 las tasas de actividad disminuyen en todos los grupos de edad. No nos parecería factible que todo ello signifique que exista un desplazamiento del hombre por la mujer en el mercado de trabajo, ya que en la mayoría de los casos se registra una separación muy marcada entre el tipo de ocupaciones que suelen realizar las mujeres y los hombres. Más bien tenderíamos a pensar que la disminución de las tasas de actividad masculina podría estar respondiendo a una sobrestimación, en la Encuesta de Hogares de la población en edad activa, posiblemente debido al planteamiento de una hipótesis de migración mayor a la que efectivamente habría tenido lugar en estos años; también podría pensarse en una frecuencia mayor de trabajadores incapacitados y de jubilaciones tempranas. En todo caso, la confirmación de estas causas requeriría de una revisión más detallada.

Esta "nueva" presencia de mujeres de mediana edad supone profundas modificaciones en los esquemas de participación y relación de ellas en los ámbitos social y familiar: nuevas pautas de 
permanencia en el mercado laboral, nuevos esquemas de conciliación de los roles de trabajadora-madre-esposa; cambios en la distribución y organización de responsabilidades familiares y de redefinición de su rol frente a la manutención del hogar.

No es posible ahondar en cada uno de esos aspectos en el marco de este trabajo, pero queda como tarea abierta. Por lo pronto, su sola mención permite ir apuntando claves de interpretación sobre el significado de los cambios en la participación de la mujer.

\section{Actividad según el estado conyugal de la mujer}

En general, se señala que la posibilidad que tienen las mujeres casadas o unidas de participar en el mercado laboral, es menor que la de las solteras y la de las viudas, separadas o divorciadas (Recchini y Wainerman, 1982; Hernández et al., 1982; Arriagada, 1990), en virtud de una mayor carga de trabajo doméstico y por la presencia de hijos, que dificultan las posibilidades de realizar actividades económicas extradomésticas.

De hecho, la participación de la mujer soltera en los países de América Latina, es mayor que la de las casadas (Elizaga, 1977; Van Roy, 1980). Sin embargo, y aun cuando todavía hoy, en la mayoría de los países latinoamericanos sigue siendo, en mucho, superior la participación de mujeres solteras, trabajos realizados en fechas recientes - por ejemplo los referidos a México (García y De Oliveira, 1990) y Argentina (Recchini de Lattes, 1982)-, apuntan que también en este aspecto estarían ocurriendo cambios significativos, evidenciándose un aumento importante en las tasas de participación de las mujeres casadas.

Para el caso de Venezuela, se señalaba a principios de los ochenta (Van Roy, 1980; Hernández, 1982) un aumento considerable del número de mujeres casadas y unidas, incorporadas al conjunto de la fuerza de trabajo, que podría "representar el comienzo de un cambio significativo respecto del estatus de la mujer en la sociedad"

Al estimar las tasas de participación según situación conyugal para 1990, de la región capital, se obtienen los resultados presentados en el cuadro 2. En primer lugar se puede observar que hay grandes variaciones en las tasas de participación de las mujeres según su estado conyugal.

Las tasas de las solteras alcanzan valores bastante elevados, pero es necesario notar la aproximación a éstas de las tasas de otras categorías conyugales. De hecho, la participación de las divorciadas es incluso superior a la de las solteras, pero este resulta- 
CUADRO 2

Venezuela, Región Capital. Tasas específicas de participación según estado conyugal por sexo, 1990

\begin{tabular}{lcc}
\hline Estado conyugal & Hombres & Mujeres \\
\hline Soltero & 54.77 & 54.10 \\
$\begin{array}{l}\text { Casado } \\
\text { (Cónyuge en el hogar) }\end{array}$ & 87.58 & 34.99 \\
$\quad$ Casado & 81.58 & 46.98 \\
$\quad \begin{array}{l}\text { (Cóny. no vive en el hogar) } \\
\quad \text { Unido }\end{array}$ & 88.23 & 32.25 \\
$\begin{array}{l}\text { (Cónyuge en el hogar) } \\
\quad \text { Unido }\end{array}$ & 73.28 & 53.33 \\
$\begin{array}{l}\text { (Cóny. no vive en el hogar) } \\
\text { Viudo }\end{array}$ & 52.17 & 34.83 \\
$\quad$ Divorciado & 76.03 & 55.74 \\
\hline
\end{tabular}

* Las tasas de participación de las mujeres fueron estandarizadas para corregir distorsiones por efecto de la estructura por edad.

Fuente: OCEI, Encuesta de Hogares por Muestreo, 1990, segundo semestre. Cálculos propios.

do no parece sorprendente, ya que las divorciadas se han mostrado siempre como un grupo de elevada participación, y cuya importancia relativa dentro del conjunto de mujeres estaría, además, incrementándose en los últimos años.

Más significativa consideramos la elevada participación de las mujeres casadas, y en particular de las unidas, cuyos cónyuges no conviven con ellas en el hogar. Dicha participación resulta muy similar a la representada por las solteras.

Estos resultados respaldan lo que ya planteábamos: en los años recientes se viene registrando un aumento notable en la participación de mujeres de mediana edad, muchas de ellas evidentemente casadas. Este incremento es resultado del rápido crecimiento de la mano de obra del grupo de mujeres casadas o unidas a partir de los tempranos sesenta. Para el total del país, entre 1961 y 1971, la cifra prácticamente se duplicó (Van Roy, 1980) y entre 1971 y 1979 creció a un ritmo de 11\% anual (Hernández et al., 1982).

Un aspecto que requiere ser destacado es que la participación de las mujeres unidas o casadas se muestra estrechamente relacionada con la presencia o ausencia habitual del cónyuge. Atendiendo a los resultados del cuadro 2 podemos observar que mientras las mujeres casadas cuya pareja reside en el hogar, tienen una tasa 
de participación de 34.99 , ésta se incrementa a 46.98 en aquellos casos en que el hombre no reside habitualmente en el hogar. En cuanto a las mujeres unidas, las diferencias que plantea esa circunstancia son todavía más amplias: 32.25 es la tasa de participación cuando el compañero está presente en el hogar y 53.33 si no lo está. Un elemento que puede incidir en el registro de esta brecha en la actividad de un tipo y otro de mujeres unidas es que la Encuesta de Hogares no distingue la oategoría de "separadas", las cuales, probablemente, quedan recogidas junto a las mujeres unidas cuya pareja no vive habitualmente con ellas.

En cualquier caso, estos resultados son muy significativos y no difíciles de entender: resulta muy claro que una mujer cuya pareja no vive con ella, lo que en muchos casos implica que no aporta dinero para el sostenimiento del hogar, se ve forzada a procurarlo ella misma. Un aspecto que requeriría, sin embargo, ser aclarado por la OCEI es por qué la Encuesta de Hogares no distingue la categoría de "separadas".

\section{Actividad según el nivel educativo de la mujer}

En cuanto a la relación entre el nivel educativo alcanzado por la mujer y su propensión a participar en actividades económicas, prácticamente todas las investigaciones realizadas coinciden en describirla como una relación positiva; la participación de la mujer en el mercado de trabajo tiende a ser más significativa en los grupos de mujeres con un mayor nivel educativo.

Sin embargo, esta relación no es determinante por sí sola y no significa, por supuesto, que la actividad laboral sólo alcance relevancia entre mujeres con cierto grado de educación. De hecho, investigaciones realizadas en México han constatado importantes aumentos en los niveles de participación de las mujeres mexicanas sin escolaridad. Un elemento fundamental es que las variaciones en la participación, según esta característica, varían sensiblemente en función del sector social: la variable educación pierde peso explicativo en aquellos grupos sociales más necesitados (García y De Oliveira, 1990).

Volviendo nuestra atención sobre Venezuela, los estudios desarrollados han afirmado repetidas veces que el incremento en la participación económica de la mujer venezolana se inserta en el proceso de masificación de la enseñanza (Valecillos, 1982; Hernández et al., 1982), fenómeno que suele ser explicado por el costo de oportunidad. Bajo tal noción se presupone que para aquellas mujeres con un mayor nivel educativo el costo de no trabajar es 
mayor, ya que pierden mejores oportunidades laborales (de remuneración más alta y más interesantes). Por lo tanto existirian presiones adicionales para optar por la alternativa de trabajar.

La mujer con un nivel relativamente bajo de educación tiene pocas esperanzas de ganar lo suficiente para poder hacer eficaz su salida diaria de la casa, por lo cual tiene mayor tendencia a quedarse en la casa permanentemente (Van Roy, 1980).

Alcanzar mayor nivel educativo significa un costo económico y personal para la mujer, por lo que ella estará más dispuesta, como contraparte, a buscar un empleo, lo cual implica remuneración y aplicación de destrezas adquiridas (Hernández, 1982).

Sin embargo, querríamos matizar este tipo de afirmaciones recordando, una vez más, que la incorporación de la mujer a actividades económicas no es función exclusivamente de su "disposición" y "expectativas"; diversos factores determinan tal realidad: personales y familiares, como los que hemos mencionado: de existencia de hijos, el número y las edades de éstos, la presencia de un cónyuge estable, y la presencia de otras mujeres en el hogar que pudiesen contribuir al cuidado de los hijos, así como la presencia de circunstancias y características sociales y económicas específicas, como los niveles salariales, las condiciones imperantes en el mercado de trabajo, y las restricciones arbitrarias que prevalecen con frecuencia, por un credencialismo exagerado.

Finalmente, hay que ser claros: las "expectativas" y el "costo de oportunidad" no son los mejores recursos explicativos para acceder a la tarea de interpretar los esquemas de participación laboral de aquellas mujeres cuya situación socioeconómica les impone, simplemente, la urgencia de trabajar para satisfacer las necesidades básicas del hogar.

Al revisar la información proporcionada por la Encuesta de Hogares para 1990, se evidencia que el patrón tradicional de mayor participación económica de las mujeres con mayor nivel educativo ha seguido prevaleciendo, claramente.

Este fenómeno respondería a diversas situaciones, pero fundamentalmente a dos: en primer lugar, a un proceso ampliamente extendido de educación masiva, que se ha desarrollado durante varias décadas, y que ha alcanzado de manera significativa a las mujeres, y en segundo lugar, a una práctica que se ha impuesto entre los sectores empleadores: la exigencia de acreditación de un nivel mínimo de escolaridad como requisito para optar a casi cualquier puesto de trabajo. Esta práctica, que caracteriza a la región capital, estaría respondiendo a una marcada especialización 
del mercado de trabajo en la misma, y por ende a un elevado nivel de competencia, mayor al que se presenta en cualquier otra región del país.

En el cuadro 3 podemos observar cómo las tasas de actividad de las mujeres aumentan a medida en que corresponden a grupos con mayor nivel educativo; las mujeres sin escolaridad o con primaria incompleta registran tasas de actividad sumamente bajas (16.3\%); en el otro extremo encontramos una altísima participación de las mujeres que tienen un nivel correspondiente a educación superior (76.03 por ciento)

CUADRO 3

Venezuela, Región Capital. Tasas de actividad según nivel educativo por sexo, 1990

\begin{tabular}{lcc}
\hline Nivel educativo & Hombres & Mujeres \\
\hline Sin escolaridad y & & \\
primaria incompleta & 70.93 & 16.36 \\
Primaria completa & 68.42 & 29.81 \\
Media y técnica & & \\
$\quad$ incompleta & 62.85 & 34.70 \\
Media y técnica & & \\
$\quad$ Completa & 76.98 & 49.39 \\
Superior incompleta & 69.65 & 48.39 \\
Superior completa & 87.42 & 76.03 \\
\hline
\end{tabular}

Fuente: ocel, Encuesta de Hogares por Muestreo 1990, segundo semestre. Cálculos propios.

Para el caso de los hombres, aunque también se observa la mayor actividad entre aquellos que cuentan con educación superior, en general, las tasas no varían tanto entre un nivel educativo y otro y, por ejemplo, los hombres del nivel de educación primaria y media o técnica registran tasas de actividad menores a la del grupo sin escolaridad o con primaria incompleta. Ello puede responder a diferencias básicas entre las actividades desarrolladas por hombres y mujeres, en el sentido de que para los primeros es más importante el componente de actividades que no requieren de mayor calificación; aunque no es posible descartar situaciones de discriminación hacia las mujeres, a las que se les exige, para una misma áctividad, mayor capacitación de la que se le podría exigir a los hombres.

Son muy significativos los incrementos que se registran en las tasas de participación cuando se pasa de un nivel sin escolaridad o de primaria incompleta a primaria completa, así como entre el nivel de educación superior incompleta y el de superior completa, lo que sin duda obedece al riguroso formalismo y credencialismo 
que impera en buena parte de los sectorés empleadores.

El análisis conjunto de la edad y del nivel educativo, y del estado conyugal con el nivel educativo resulta útil para confirmar la consistencia de los efectos que estarían ejerciendo tales variables sobre las posibilidades de participación de la mujer (véanse cuadros 4 y 5 ).

\section{CUADRO 4}

Venezuela, Región Capital. Tasas de actividad femeninas por grupo de edad según el nivel educativo, 1990

\begin{tabular}{lccccccc}
\hline Nivel educativo & $15-19$ & $20-24$ & $25-29$ & $30-34$ & $35-39$ & $40-44$ & $45-49$ \\
\hline $\begin{array}{l}\text { Sin escolaridad y } \\
\text { primaria incompleta }\end{array}$ & 18.97 & 27.50 & 31.52 & 40.00 & 46.88 & 40.63 & 39.53 \\
$\begin{array}{l}\text { Primaria completa } \\
\begin{array}{l}\text { Media y técnica } \\
\text { incompleta }\end{array}\end{array}$ & 24.77 & 36.53 & 40.08 & 45.61 & 45.71 & 46.61 & 47.16 \\
$\begin{array}{l}\text { Media y técnica } \\
\text { completa }\end{array}$ & 14.48 & 44.22 & 46.76 & 54.44 & 52.29 & 60.35 & 53.85 \\
$\begin{array}{l}\text { Superior incompleta } \\
\text { Superior completa }\end{array}$ & 22.07 & 58.37 & 61.27 & 68.16 & 67.52 & 59.74 & 55.71 \\
& 6.67 & 34.24 & 66.61 & 64.96 & 68.35 & 68.33 & 55.88 \\
\end{tabular}

Fuente: OCEI, Encuesta de Hogares por Muestreo, 1990, segundo semestre. Cálculos propios.

Si se calculan las tasas de actividad de las mujeres entre 15 y 49 años, distinguiendo simultáneamente tanto el grupo de edad como el nivel educativo, es posible observar que, al interior de cada grupo de edad, permanece la tendencia creciente de participación económica de la mujer en.la medida en que es mayor su nivel educativo (véasé cuadro 4). La lectura del mismo cuadro en un sentido horizontal muestra también la tendencia, para mujeres con un mismo nivel educativo, a participar más en la medida en que aumenta la edad, hasta alcanzar un máximo entre los 30 y 44 años, a partir de lo cual, la participación tiende a descender significativamente; se diferencia en este sentido el grupo de mujeres con primaria completa cuya participación se mantiene en aumento hasta los 49 años, aunque es probable que descienda en una edad posterior.

Al revisar de manera conjunta el comportamiento de las tasas según el nivel educativo y el estado conyugal de las mujeres (véase cuadro 5), una vez más se evidencian, a grandes rasgos, mayores tasas de participación para las mujeres de un mismo estado conyugal en la medida en que aumenta su nivel educativo. Las mujeres solteras constituyen en este aspecto la excepción, ya que se obtiene una mayor tasa de participación para las solteras sin escolaridad y con primaria que para las solteras con educación 


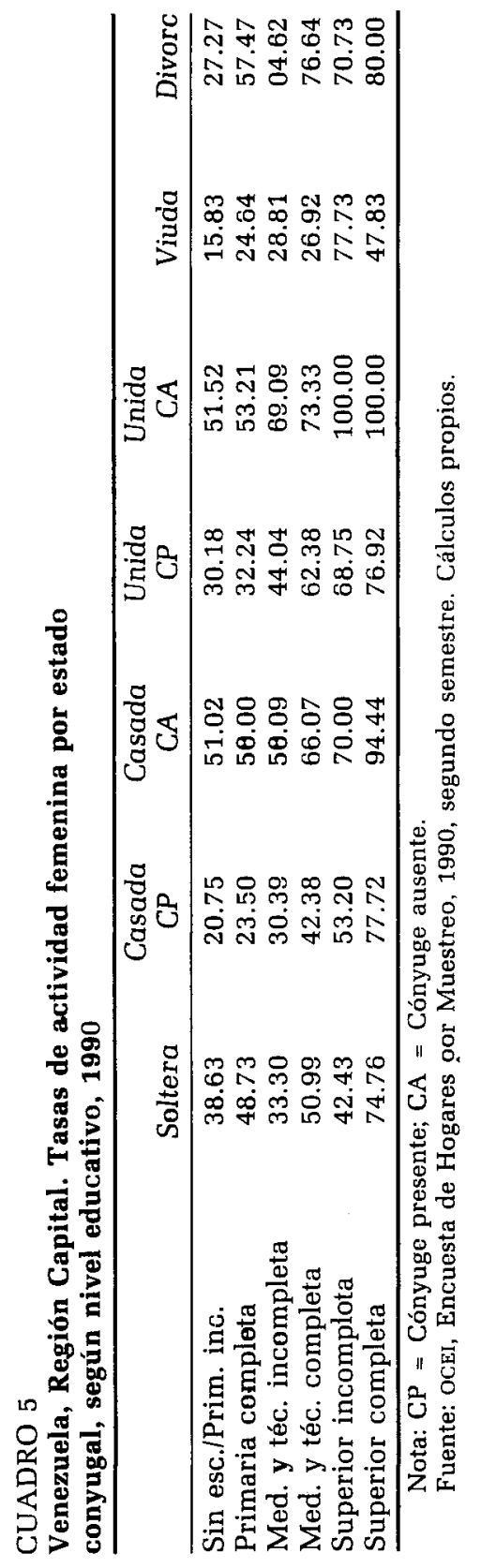


media y técnica incompleta; sin embargo, la caída en las tasas de participación de esta categoría es bastante fuerte, lo que nos hace pensar que puede deberse a problemas en la Encuesta.

Un aspecto interesante que nos confirma este último cuadro es que las tasas de participación de las mujeres casadas y unidas con cónyuge ausente son, casi invariablemente, las más altas en cada nivel educativo; se puede observar que aún las tasas de participación de estas mujeres sin nivel educativo corresponden a valores que las otras mujeres sólo alcanzan con un nivel educativo medio o técnico.

Tal situación nos permite reafirmar que la influencia de la educación sobre las pautas de participación pasa necesariamente por la mediatización de otros aspectos y características que afectan a la mujer y frente a los cuales aquélla puede llegar a perder capacidad determinante.

\section{Comentario final}

Los resultados encontrados apuntan, de manera clara, la presencia de transformaciones en el patrón por edad y estado conyugal de la mujer trabajadora promedio. Durante la década pasada tuvo lugar una intensificación de la participación de mujeres de mediana edad, con el desplazamiento de la mayor tasa de actividad del grupo de 25-34 años al grupo de 35-44 años. De igual manera, el incremento en la participación femenina durante este periodo estuvo ligado a una participación más significativa de las mujeres casadas o unidas, particularmente de aquellas que no conviven con su pareja.

¿Por qué esta "nueva presencia" en el mercado de trabajo de mujeres que quedaban excluidas por su situación conyugal, familiar, o por su edad? Para exlicarlo nos vemos remitidos, forzosamente, a suponer la presencia de elementos o condiciones diferentes que han promovido esta participación más intensa.

Pero no se trata de una causa. En el proceso convergen aspectos de distinta naturaleza, que van desde posibles modificaciones en la demanda de mano de obra, menor tamaño de la familia, más baja fecundidad, culminación más temprana de la reproducción, hasta cambios en las motivaciones personales de la mujer.

Un elemento al que podría atribuirse responsabilidad en el fenómeno es el que constituyen los cambios en las estructuras e instituciones sociales, que estuviesen facilitando la permanencia de la mujer en el mercado de trabajo, a pesar de las importantes cargas familiares que muchas de ellas deben asumir. Indudablemente 
en el caso de nuestro país, la presencia y efectos de elementos de esta naturaleza es muy reducida; aunque se han desarrollado algunos programas, estamos todavía muy lejos de poder afirmar que existe una infraestructura de servicios y programas que actúen decisivamente como facilitadores de la doble jornada de trabajo que enfrenta el común de las mujeres.

Por otra parte, algunos cambios sociales y culturales, a favor de una mayor apertura y valoración de la mujer trabajadora, han tenido cierta incidencia. Ellos implican la modificación, en alguna medida, de juicios y actitudes frente a la mujer casada, probablemente con hijos, que trabaja, y que debe delegar sus responsabilidades en el hogar o de algún modo compatibilizarlas con su actividad económica; con certeza, el impacto ha sido más determinante en la región capital que en cualquier otra ciudad o región del país, al constituir aquélla la puerta fundamental de intercambio y recepción de nuevas pautas y valores, y por tanto un espacio privilegiado para la consolidación de nuevos procesos sociales. Pero, aunque tales elementos culturales han permitido catalizar el proceso de incorporación de la mujer al escenario laboral, éstos no resultan suficientes por sí solos para explicar los cambios presentes respecto a las características de la mujer trabajadora.

También creemos que es importante destacar el papel preponderante que la crisis ha tenido en todo este proceso, acelerándolo: la difícil situación económica del país se ha traducido necesariamente en la búsqueda de nuevas alternativas de sobrevivencia en los hogares venezolanos. Y en estas "nuevas estrategias", el aporte que la mujer viene haciendo y puede hacer es fundamental.

\section{Bibliografía}

Anker, Richard y Ruth Dixon-Mueller (1989), Evaluación del aporte económico de la mujer al desarrollo, Ginebra, Oficina Internacional del Trabajo.

Arriagada, Irma (1990), "La participación desigual de la mujer en la fuerza de trabajo", en Revista de la CEPAL, núm. 40, abril, Santiago de Chile, pp. 87-104.

Cartaya, Vanessa (1986), Empleo e ingresos en Venezuela: situación actual, perspectivas y alternativas, Caracas, Instituto Latinoamericano de Investigaciones Sociales (ILDIS).

Christenson, Bruce, Brígida García y Orlandina de Oliveira (1989), "Los múltiples condicionantes del trabajo femenino en México", en Estudios Sociológicos, vol. vil, núm. 20, México, El Colegio de México, pp. 179-251.

Elizaga, Juan Carlos (1977), Participación de la mujer en la mano de obra 
en América Latina: la fecundidad y otros determinantes, Serie D, núm. 95, Santiago de Chile, Celade.

García, Brígida y Orlandina de Oliveira (1990), Recesión económica y cambio en los determinantes del trabajo femenino, México, El Colegio de México (mimeo.).

Hernández, Marisela, Delia Lanz y María Digna Iglesias (1982), “Características del mercado laboral femenino en Venezuela", ponencia presentada en la Primera Jornada el Trabajo y la Productividad de la Mano de Obra Femenina, Caracas, Ministerio de Estado para la Participación de la Mujer en el Desarrollo, febrero, $30 \mathrm{pp}$.

Mazzei, Milena (1986), "Población y empleo: Venezuela, evolución de la población económicamente activa y estado actual de la investigación 1981-1985", ponencia del Encuentro Nacional de Demógrafos y Estudiosos de la Población, Estado Actual de los Estudios de la Población en Venezuela, Caracas, IIES-UCAB-OCEI-nLDIS, septiembre.

Pantelides, Edith (1976), Estudio de la población económicamente activa en América Latina, 1950-1970, Santiago de Chile, Celade.

Recchini de Lattes, Zulma y Catalina Wainerman (1981), El trabajo femenino en el banquillo de los acusados. La medición censal en América Latina, México, The Population Council.

(1982), "La temática del trabajo femenino: contribuciónes a su explicación”, en Estudios sobre la Mujer, México, Secretaría de Programación y Presupuesto, pp. 73-95.

Valecillos, Héctor (1982), "Evolución histórica, situación actual y perspectivas del trabajo de la mujer en Venezuela", ponencia presentada en la Primera Jornada el Trabajo y la Productividad de la Mano de Obra Femenina, Caracas, Ministerio de Estado para la Participación de la Mujer en el Desarrollo, febrero, $20 \mathrm{pp}$.

Van Roy, Ralph (1980), "La participación de la mujer en la fuerzá de trabajo: el caso de Venezuela", en Revista sobre Relaciones Industriales y Laborales, Caracas, Universidad Católica Andrés Bello, abril-junio, pp. 17-40. 